\title{
Effect of Temperature on Physical Properties of Canadian Maple (Acer saccharum marsh) Syrup
}

\author{
Marta A. Pires ${ }^{1}$, Rebecca S. Andrade ${ }^{2}$, Miguel Iglesias ${ }^{3 *}$ \\ ${ }^{1}$ Maurício de Nassau University, Department of Nutrition, Salvador, Bahia; ${ }^{2}$ Recôncavo da Bahia Federal University, \\ Technology Science Center in Energy and Sustainability, Feira de Santana, Bahia; ${ }^{3}$ Federal University of Bahia, \\ Department of Chemical Engineering, Salvador, Bahia, Brazil
}

The optimization of industrial operations requires knowledge of thermodynamics related to the process, which can be determined either experimentally or by predictions based on an appropriate model and a set of data. Although maple syrup is a nearly unprocessed natural product, its industrial manufacture applies usual chemical-mechanical fluid operations. So, appropriate equipment designs are conditioned by sufficient information on mixing thermodynamics. In this paper, we analyze the temperature effect on maple syrup and its aqueous dilution in terms of different properties, trying to explain their special physicochemical behavior to explore the strength of the interactions among heavy covalent macromolecules and shorter chain polar solvents. Keywords: Food Engineering. Canadian Maple Syrup. Thermodynamic Properties. Theoretical Model.

Abbreviations: P: density; u: ultrasonic velocity; Lf: free length; $Z$ : specific acoustic impedance; b: van der Waals' constant; S: collision factor.

\section{Introduction}

Canada produces more than 78 percent of the world's maple syrup. In 2016, Canadian producers exported 45 million $\mathrm{kg}$ of maple products, with a value of US\$381 million. Canada's share of the world's maple production increased over $225 \%$ in the last decade, exporting to more than 50 countries. The most important export market is the United States, to which Canadian producers send $65 \%$ of total exports. Other principal markets are Germany (11\%), Japan (7\%), United Kingdom (4\%), Australia (4\%), and France (4\%). The chemical complexity of the maple syrup taste is not completely known yet. Flavor compounds of maple syrup include volatile phenolic compounds, carbonyl compounds, and alkyl pyrazines [1], typical products of the advanced stages of the Maillard reaction, which have been the subject of numerous studies because of the impact on the flavor and color of different foods [2]. The most

Received on 10 September 2020; revised 21 December 2020. Address for correspondence: Miguel Iglesias. Federal University of Bahia. Rua Aristides Novis, 2, Federação. Zip Code: 40210-630 Salvador, Bahia, Brazil. Phone: (5571)3283-9805. E-mail: miguel.iglesias@ufba.br. Article selected from VI International Symposium on Innovation and Technology (SIINTEC).

J Bioeng. Tech. Appl. Health 2021;4(1):30-36.

(C) 2021 by SENAI CIMATEC. All rights reserved. widely accepted mechanism for the formation of pyrazines in food systems is via the Strecker degradation of amino acids, which in the presence of a-diketones result in the formation of a -aminoketones and Strecker aldehydes [3]. The formation of pyrazine compounds is considered to require sugar fragments. It has been reported that alkaline conditions promote sugar fragmentation and result in increased formation of pyrazines. These compounds, mostly found in heated foods, have organoleptic characteristics [4]. Different aminoacids [5] and sucrose, glucose, or fructose [6-7] present in the maple sap are the main precursors of the pyrazines in maple syrup. Maple syrup is considered a better alternative to traditional refined sugar and many other available natural sweeteners, due to its oligo-elements profile and high amount of bioactive compounds with well-known antioxidant potential action [8-10]. Despite the economical and nutritional importance of this natural product, only a few studies in the last years have been developed closely related to chemical determination [1112], the application of instrumentation techniques for quality control, adulteration, and compounds identification [11, 12] or treatment procedures. Most of them are related to narrow operational conditions and the chemical constitution of maple sap or syrup, but not with thermophysical characteristics, their temperature dependence, or 
potential applications in industrial elaboration. Regarding the unit operation field, optimization of industrial operations requires knowledge of the thermodynamics of compounds and mixtures related to the process, which can be determined either experimentally or by predictions based on an appropriate model and a set of data. Although maple syrup is a nearly unprocessed natural product, its industrial manufacture applies usual chemical and mechanical fluid operations, so the optimization and adequate designs of equipment are conditioned by sufficient knowledge of mixing thermodynamics. Operations such as pumping, evaporation, or filtration are required for the sap treatment and for quality control during the process, which is based on the evolution of physical properties of the sap throughout the process. Refractive techniques are usually applied to determine the mature point of evaporation and the quality of the maple syrup, although no bibliographic data has been found in the open literature.

In this paper we analyze the temperature effect $(278.15-323.15 \mathrm{~K})$ on the maple syrup and aqueous dilution of this product, in terms of density and isentropic compressibility, assuming that in the last steps of maple syrup concentration no significant chemical changes take place and only water is removed. We have attempted to explain the physicochemical behavior of the mixtures indicated above, to explore the strength and nature of the interactions among the complex components evolved.

\section{Material and Methods}

Due to the importance of theoretical knowledge on industrial design, the prediction of physical property values was realized by applying different methods. For density, a modified equation of Subbiah-Barber, and an empirical equation for ultrasonic velocity. The obtained results were analyzed and commented upon. Various parameters such as intermolecular free length (Lf), specific acoustic impedance (Z), van der Waals'constant(b), collision factor $(\mathrm{S})$, and compressibility hydration number (nh) were computed. The analysis of these volumetric and acoustic magnitudes pointed out the availability of intense effects among solute + solvent molecules at a determined range of concentration and temperature. Attending to the deviation in computed data, we conclude that the application of these models shows close agreement with the experimental data reported in this paper. The present study of thermodynamics shows the strong dependence of these properties on temperature and the amazing trend of the isotherms, showing them as an accurate alternative procedure for determining the optimal point of syrup concentration by sap evaporation.

\section{Results and Discussion}

Maine law requires maple syrup to be evaporated to a density greater than $66 \%$ Brix at 68 degrees F. Parenthetically, syrup with a density reading below $66 \%$ Brix is illegal since it is more likely to ferment. With density above $68 \%$ Brix, it may crystallize, causing consumer complaints. Various instruments can be used to check the density: hydrometers, hydrothermal, refractometers, light transmittance meters, and many others. While the particular grade of pure maple syrup is largely determined by color, all grades of syrup must meet minimum density standards. In most maple syrup producer countries, the minimum allowable density of maple syrup is $66 \%$ by weight of soluble solids ( 66.0 degrees Brix at 68 degrees F). Although syrup density can be measured in three ways - (1) weight, (2) use of an optical refractometer and (3) use of a hydrometer -, only the refractometer and hydrometer methods are recommended for producer use. Syrup weight may be used as an estimate of volume, but it is too imprecise to be used as an indicator of density. While the minimum syrup density of 66.0 degrees Brix is a legal requirement in most states, there are also several practical reasons for carefully controlling the finished density of maple syrup. Viscosity, a measure of a fluid's resistance to flow, 
is an important characteristic of maple syrup. Until the sugar concentration of maple sap exceeds 30 degrees Brix, an increase in sugar percentage has relatively little effect on viscosity. However, as the sugar concentration increases toward and through that of standard density syrup, the increase in viscosity is more pronounced. Maple syrup has a density only 0.5 degrees to 1 degree Brix below standard density syrup feels and tastes thin. Conversely, an increase of only 1 degrees Brix above standard density causes the syrup to acquire a thick, pleasant feel to the tongue, and the perception of considerably increased sweetness. This explains why some producers finish their syrup slightly above standard syrup density; customers can tell the difference between 66 degrees and 67 degrees Brix and prefer the heavier syrup. Syrup density also affects how well quality is maintained when the syrup is stored. Light density syrup spoils faster. Syrup with a density of more than 67 degrees Brix may precipitate sugar crystals when stored at room temperature for extended periods. Finally, the higher the density at which syrup is finished, the less that can be made from a given amount of sap. Using a refractometer to determine the density of syrup by measuring its refractive index is a relatively accurate, yet simple method. This possibility has been checked in earlier works for another kind of mixtures. A refractometer works by measuring the refractive index of a solution (syrup) which is directly related to the number of dissolved solids (sugar) present in the solution. Refractometers are precise instruments and are particularly well suited for determining the density of syrup in Brix units at room temperatures. They are not well suited for measuring the density of hot syrup (180 degrees $\mathrm{F}$ and above) but are very convenient for larger operations of buying and selling syrup. They are also commonly used to determine the density of syrups entered in various competitions. But as far as we know, there is no open literature data of refraction for maple syrup taking into account this magnitude is strongly dependent on temperature. This fact potentially is the cause of wrong determination of the optimal point of dehydration, due to inaccurate data of refraction to translate experimental measurements and inaccuracy in controlling the temperature of samples to be measured. Moreover, the computation of density through refraction leads to errors in the final density values. To avoid these problems, it is necessary the use direct magnitude measurements and the disposable of its temperature dependence. The measured physical properties were correlated as a function of temperature using Eq. 1:

$$
P=\sum_{i=0}^{N} A_{i} T^{i}
$$

in which $\mathrm{P}$ is density $\left(\mathrm{gcm}^{-3}\right)$ or ultrasonic velocity $\left(\mathrm{ms}^{-1}\right), \mathrm{T}$ is the absolute temperature in Kelvin and $\mathrm{Ai}$ are fitting parameters. $\mathrm{N}$ stands for the extension of the mathematical series, optimized through the Bevington test. The fitting parameters were obtained by the unweighted least squared method applying a fitting Marquardt algorithm. The root means square deviations were computed using Eq. 2, where $\mathrm{z}$ is the value of the property, and nDAT is the number of experimental data.

$$
\sigma=\left(\frac{\sum_{i=1}^{n_{\mathrm{DAT}}}\left(z_{\mathrm{exp}}-z_{\mathrm{pred}}\right)^{2}}{n_{\mathrm{DAT}}}\right)^{1 / 2}
$$

In Figures $1 \mathrm{a}$ and $1 \mathrm{~b}$, the temperature trend of density and ultrasonic velocity are gathered.

We have attempted to explain the physicochemical behavior of this mixture, to explore the strength and nature of the interactions between the components by deriving various thermodynamic parameters from the new collection of density and ultrasonic velocity. The parameters derived from the experimental measured data were intermolecular free length (Lf), specific acoustic impedance (Z), van der Waals' constant (b), and collision factor (S). As observed, intermolecular free length decreases 
Figures 1a and 1b. Effect of temperature for (a) density and (b) ultrasonic velocity of Canadian maple syrup + water.

a)

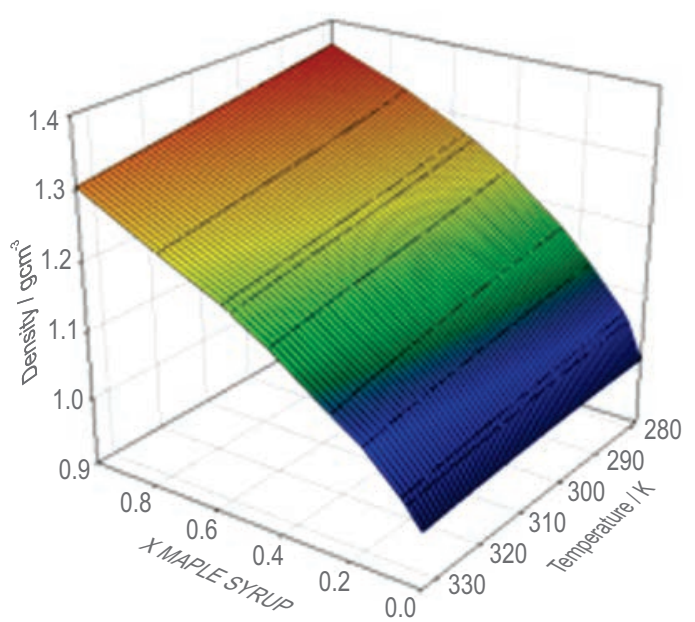

$\mathrm{x}$ : molar fraction of maple syrup.

from pure solvent (water) towards pure solute (maple syrup), showing the lowest values for low temperatures, as expected. While van der Waals constant gathers a linear performance in terms of composition or temperature effect, the specific acoustic impedance and collision factor show an inverse trend of the enclosed pure substances, mainly due to the nature of the complex collection of covalent molecules enclosed into the "maple syrup concept". That goes to show how much resistance an ultrasound beam encounters as it passes through the liquid phase, or, in other words, as the probability of collision among molecules rises for high concentration compositions and low temperatures.

Despite solvation numbers of covalent molecules into the aqueous solution being key parameters necessary when discussing intermolecular interactions or interfacial phenomena, this data is extremely scarce and dispersed into open literature. If the search for such data was related to a solvent other than water, the situation is worse. Solvation numbers are based on isentropic compressibilities (computed by the Newton-Laplace equation from density and ultrasonic velocity), a parameter easily derived from acoustic measurements, as previously commented. The compressibility solvation numbers, or compressibility hydration numbers for an aqueous b)

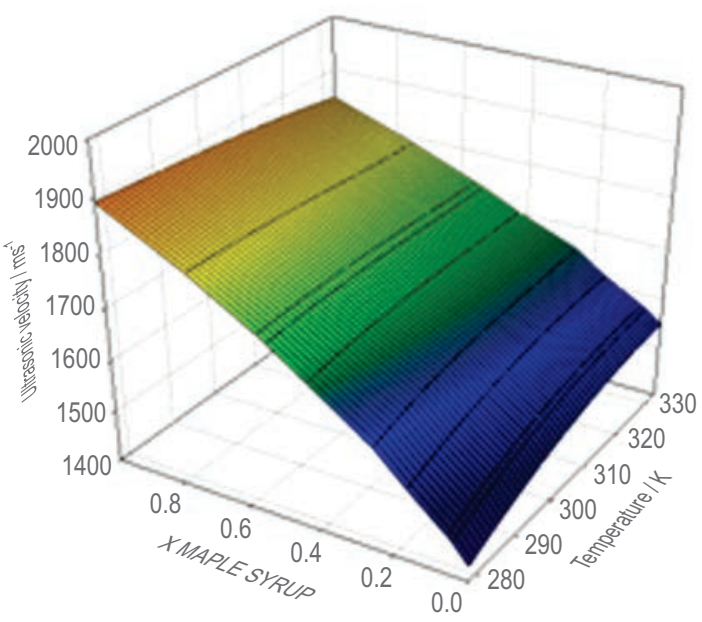

environment, are calculated using the following equation, attending to the usual expressions:

$$
n_{S}=\left(\frac{n_{\text {solvent }}}{n_{\text {solute }}}\right) \cdot\left(1-\frac{\kappa_{\mathrm{S}}}{\kappa_{\mathrm{So}}}\right)
$$

in which nsolvent and nsolute are the mol number of solvent and solute into a binary mixture, respectively. The equation used for computing compressibility hydration number assumes that the solvation layer around the corresponding solute molecule is incompressible, which is not the case. Despite this, it provides an acceptable approximation of the extent of interaction of the solute or solutes with solvent. These parameters are derived from isentropic compressibility measurements and therefore account for the first two layers of solvent around the solute. For example, Figure 2 shows the evolution of the compressibility hydration number for the mixture maple syrup + water as a function of temperature, gathering the strong diminution of disposable solvent molecules for the establishment of layers around each theoretical solute molecule. As observed, only a slight effect is produced by variation of temperature at the studied range. When the temperature is increased, there is a corresponding decrease in the ultrasonic velocity for concentrated solutions, and then an increment of the entropy 
Figure 2. Effect of temperature for compressibility hydration number of Canadian maple syrup + water.

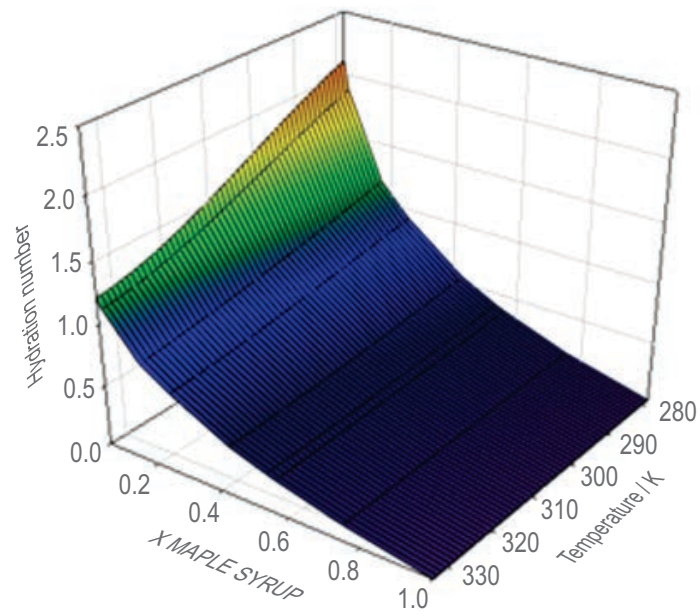

x: molar fraction of maple syrup.

of the system, the strongest values of solvation numbers being observed for low temperatures. The physical property packages used in powerful chemical simulators typically rely on generalized equations for predicting properties as a function of temperature, pressure, etc. In the last few years, despite the success of developing several procedures of density estimation for pure compounds or mixtures, only a few of them may be of practical application and high accuracy for fats and oils. The procedure proposed by Subbiah et al. for the description of the density of aqueous sucrose using a polynomic expansion has been proven accurate, only requiring a description of pure sucrose density dependence with temperature as suggested by Barber:

$$
\begin{aligned}
& 1 / \rho=\sum_{i=1}^{N} \frac{w_{i}}{\rho_{i}} \\
& \rho_{\text {sucrose }}=a+b \cdot w_{\text {sucrose }}+c \cdot w_{\text {sucrose }}^{2} \\
& a=1662.7-2.5025 \cdot T+0.0306 \cdot T^{2} \\
& b=-57.953+2.2511 \cdot T-0.0417 \cdot T^{2} \\
& c=-40
\end{aligned}
$$

in which $r$ is the density of the mixture, and wi is the mass fraction of the mixture.
Ultrasonic velocity has been systematically measured in the last years but this kind of thermodynamic data is still extremely scarce for biological solutions, specifically for food engineering. The experimental data were compared with the values obtained by the Junjie equation, which is dependent on the values of density and ultrasonic velocity of each component into the mixture:

$$
u=\frac{\sum_{i=1}^{N}\left(x_{i} M_{i} / \rho_{i}\right)}{\left(\sum_{i=1}^{N}\left(x_{i} \cdot M_{i}\right)\right)^{1 / 2}\left(\sum_{i=1}^{N}\left(x_{i} M_{i} /\left(\rho_{i}^{2} u_{i}^{2}\right)\right)\right)^{1 / 2}}
$$

in which $\mathrm{Mi}$ is the molar mass of each component and $\mathrm{xi}$ is the molar fraction composition. Table 1 shows the deviation of these estimation methods.

Maple syrup and sugars during refining are examples of complex solutions in a high polar environment. The physical properties of such systems are necessary for an adequate design and optimization of processes and a deeper understanding of the behavior of the final product. Literature shows limited disposable data and even less rigorous analysis of the physical properties of maple syrup. A fundamental thermodynamic approach provides an effective basis for the analysis and prediction of these properties. 
Table 1. Deviation of the estimation methods for density and ultrasonic velocity for the mixture Canadian maple syrup + water at $298.15 \mathrm{~K}$.

\begin{tabular}{lcc}
\hline Mixture & Subbiah-Barber equation & Junjie equation \\
\hline Maple Syrup + Water & $0.18693 \mathrm{gcm}^{-3}$ & $33.81 \mathrm{~ms}^{-1}$ \\
\hline
\end{tabular}

The main focus of this study was to increase volumetric and acoustic property data as a function of temperature for aqueous solutions of Canadian maple sugar graded as Canada Grade A Golden (delicate taste, $\geq 75.0 \% \mathrm{~T}$ ), former Canada \#1, Extra Light, compute derived thermodynamic properties and analyze the accuracy of theoretical empirical models for predicting data.

\section{Conclusion}

From the experimental investigation and the above discussions, the following conclusions have been drawn:

1. The tested methods Subbiah-Barber equation (SBE), and the Junjie equation (JE) showed the accurate capability of prediction of the measured magnitudes at the range of application, especially for low compositions, despite their empirical character and complexity of the studied solutions. These models have better predictive capacity at low temperatures for density, and high temperatures for ultrasonic velocity. The consideration of maple syrup as a concentrated solution of sucrose was a simplification, but the applied model deviations were acceptable.

2. A review of volumetric and acoustic properties of Canadian maple syrup revealed an important gap in disposable open literature thermodynamic data, despite its economic importance and its strong influence as a necessary tool for the accurate establishment of the optimal point of thermal operation, the gathered data being a new contribution of consistent technical information for industrial maple operators.

\section{Acknowledgments}

Miguel Iglesias carried out sample preparation and experimental measurement of properties. Rebecca S. Andrade and Marta A. Pires performed the data processing. All the authors collaborated in the preparation of the final manuscript. The authors would like to acknowledge the National Council for Scientific and Technological Development - CNPq (M. Iglesias - Chamada CNPq No 29/2019, Processo 313601/2019-4 and R. S. Andrade - Chamada MCTIC/CNPq $N^{o}$ 28/2018, Processo 438376/2018-8) for its support in developing this research.

\section{References}

1. Belford AL, Robert CL, Stephen CR. Contributions of selected flavor compounds to the sensory properties of maple syrup, Journal of Sensory Studies. 1991;6(2):101-118.

2. Maga JA, Sizer CE. Pyrazines in foods. A review. Journal of Agriculture and Food Chemistry 1973;21:22-30.

3. Hwang HI, Hartman TG, Rosen RT, Lech J, Ho C. Formation of pyrazines from the Maillard reaction of glucose and lysine-R-amine-15N, Journal of Agriculture and Food Chemistry 1994;42:10001004.

4. Fors S. Sensory properties of volatile Maillard reaction products and related compounds: A literature review. In The Maillard Reaction in Foods and Nutrition; Waller G, Feather MS, Eds.; ACS Symposium Series; American Chemical Society: Washington, DC. 1983;215:185-286.

5. Kallio H. Comparison and characteristics of aroma compounds from maple and birch syrup in Proceedings, 5th Int. Flavor Conf. (G. Charalambous, ed.) p. 241, Elsevier, Amsterdam, 1988.

6. Jones ARC, Alli I. Sap yields sugar content, and soluble carbohydrates of saps and syrup of some Canadian birch and maple species. Canadian Journal for Forest Research 1987;17:263-266. 
7. Leech RH, Kim YT. Methods to investigate fertilization as a means to improve growth and sugar yield of sugar maple. Communications in Soil Science and Plant Analysis 1990;21:2029-2039.

8. Phillips KM, Carlsen MH, Blomhoff R. Total antioxidant content of alternatives to refined sugar. Journal of the American Dietetic Association 2009;109(1):64-71.

9. González-Sarrías A, Li L, Seeram NP. Anticancer effects of maple syrup phenolics and extracts on proliferation, apoptosis, and cell cycle arrest of human colon cells. Journal of Functional Foods 2012;4(1):185-196.
10. Mellado-Mojica E, Seeram NP, López MG. Comparative analysis of maple syrups and natural sweeteners: Carbohydrates composition and classification (differentiation) by HPAEC-PAD and FTIR spectroscopy-chemometrics. Journal of Food Composition and Analysis 2016;52:1-8.

11. Filipic VJ, Underwood JC, Dooley CJ. Trace Components of the Flavor Fraction of Maple Syrup, Journal of Food Science 1969;34(2):105-110.

12. Underwood JC. Effect of heat on the flavoring components of maple sirup, Journal of Food Science. 1971;36(2):228-230. 